\title{
EL ENFOQUE STEAM: DISEÑO PARTICIPATIVO EN UNA EXPERIENCIA DE CIENCIA CIUDADANA
}

\author{
Francisco Javier Serón Torrecilla \\ Universidad de Zaragoza. Dpto Didáctica de las Ciencias Experimentales \\ Escuela Superior de Diseño de Aragón. Dpto. Fundamentos Científicos de Diseño
}

\begin{abstract}
Resumen
La creación de objetos es una actividad artística y de diseño que permite un trabajo desde enfoques y metodologías diversas. Cada vez más, se observan propuestas vinculadas a una sociedad de conocimiento abierto que incorpora espacios multi-agente. En el presente artículo se presenta y analiza un proyecto, que bajo el enfoque STEAM, intersecta la dimensión artística y de diseño, con el concepto de ciencia ciudadana, y en el que, junto a nuestros estudiantes del Grado en Diseño de Producto, han intervenido en la solución del problema numerosos agentes. En la introducción se situa el contexto social y educativo para la intervención. Se continua con una revisión teórica de las bases de un diseño abierto y coparticipado en su imbricación con experiencias de ciencia ciudadana, señalando algunos aspectos en relación al enfoque metodológico. En el siguiente apartado se apuntan los objetivos generales de la propuesta en relación al diseño del artefacto, así como su descripción. Por último se detallan los resultados desde un marco reflexivo y la coherencia con el enfoque analizado. Para finalizar se apuntan unas breves conclusiones sobre la dimensión cognitiva que movilizan este tipo de propuestas y su articulación en torno a la metodología empleada.
\end{abstract}

\section{Palabras Claves: DISEÑO DE CIENCIA CIUDADANA; STEAM; DISEÑO; ARTE; EDUCACIÓN EN DISEÑO}

\section{THE STEAM APPROACH: PARTICIPATORY DESIGN IN A CITIZEN SCIENCE EXPERIENCE}

\section{Abstract}

The creating of objects is an artistic and design activity that allows a work from diverse approaches and methodologies. Increasingly, we see proposals associated with an open knowledge society that incorporates multi-agent practices. This article presents and analyses a project, which under the STEAM approach, intersects the artistic and design dimension with the concept of citizen science, and in which, together with our students from the Degree in Product Design, numerous agents have intervened in the solution of the problem. In the introduction, the social and educational context for the intervention is placed. It continues with a theoretical review of the principles of an open and co-participated design in its overlapping with experiences of citizen science, pointing out some aspects in relation to the methodological approach. In the following section, the general objectives of the proposal in relation to the design of the artefact are pointed out, as well as its description. Finally, the results are detailed from a reflective framework and the coherence with the analyzed approach. Finally, some brief conclusions about the cognitive dimension that motivate this kind of proposals and their articulation around the methodology used are noted.

\section{Keywords: CITIZEN DESIGN SCIENCE; STEAM; DESIGN; ART; DESIGN TRAINING}

\footnotetext{
Serón Torrecilla, Francisco Javier. 2020. "El enfoque STEAM: Diseño participativo en una experiencia de ciencia ciudadana". AusArt 8 (1): 247-257. DOI: 10.1387/ausart.21474
} 


\section{INTRODUCCIÓN}

Las sociedades abiertas requieren, para ser denominadas de tal forma, la continua participación de la ciudadanía en la toma de decisiones y en la construcción del conocimiento. Configurando de esta forma estrategias de horizontalidad más equilibrada acordes a las distitnas perspectivas y voluntades.

La formación en diseño sigue en general desarrollándose según procesos estancos en los que se involucra muy poco esa toma de decisiones horizontal en el que interviene la sociedad en la búsqueda de soluciones a sus problemas; uno de los principios fundamentales del diseño social desde los años setenta del siglo veinte.

En el ámbito de la creación en, y desde el diseño, así como del arte, con la llegada de la tecnología se ha producido una cada vez más y mayor democratización en las producciones. Es, este marco de cambio social, en el que el enfoque STEAM (Science, Technology, Engineering, Art-[Arte+Diseño], Mathematics) no solo cobra un mayor sentido, dentro de su todavía relativa novedad, sino que se expande hacia terrenos por explorar. El proyecto aquí presentado nace de la necesidad de romper el aislamiento formativo y social de nuestros estudiantes de diseño. Se presenta con la finalidad de promover la integración cognitiva racional e intuitiva de la propia dimensión diseño, recogiendo de este modo, a nuestro entender, muchos de los principios que subyacen al propio enfoque de trabajo STEAM: coperativo, co-creado, coparticipado, transdisciplinar.

En el artículo se aborda un proceso de diseño creativo participado bajo un nuevo paradigma de generación de conocimiento, como es la denominada ciencia ciudadana y lo que autores del ámbito anglosajón han denominado ciencia del diseño de la ciudadanía (CDS por sus siglas en inglés) o diseño participativo de experiencias de ciencia ciudadana en castellano. Un proceso concebido como un espacio de cooperación de múltiples agentes que en este caso involucra a: estudiantes de diseño de producto de la Escuela Superior de Diseño de Aragón (en adelante ESDA) voluntarios expertos en electrónica abierta (Arduino) e implementación del interntet de las cosas (IOT por sus siglas en inglés), expertos en zoología y biología de especies urbanas, centros como Etopía-CAT (centro Etopía para el arte y la tecnología por su denominación en castellano) y estudiantes de primaria, así como sus maestros y maestras. 
Participantes que presentan distintos objetivos, competencias y necesidades, pero qu e han compartido un objetivo común, analizar y buscar solución a una problemática del ecosistema urbano: la mayor presencia de insectos y su incidencia en un problema de salud pública en la ciudad de Zaragoza.

De esta forma, y como experiencia de ciencia ciudadana, se ha articulado, a partir de la dimensión artística y de diseño, un espacio de aprendizaje en el uso de las tecnologías, la nueva ingeniería de la información abierta y el bigdata bajo los principios metodológicos STEAM. El escrito presentado, dada su extensión, se ha centrado en señalar las líneas que conectan el trabajo de los estudiantes de diseño a partir de la elaboración del artefacto en el marco de un proyecto más global, poniendo de manifiesto cómo la dimensión artística y de diseño, bajo este enfoque, permite abordar propuestas que no solo impactan socialmente, sino que permiten una mejora en la formación de los propios estudiantes, alterando o introduciendo nuevos roles, en coherencia como se apuntará en el marco teórico con lo señalado por algunos autores.

\section{MARCO TEÓRICO}

La naturaleza del enfoque STEAM, desde su propia conceptualización, incorpora aquellos elementos provenientes de la dimensión más creativa del arte y sobre todo del diseño (Maeda 2013) aunque este último, quede oculto en la propia -A del acrónimo. Las bases históricas del diseño como una multidisplina proyectual, así como las metodologías que le son propias, presentan múltiples puntos en común con lo que es el trabajo por proyectos en su adecuación al propio enfoque metodológico (Azcaray 2019), y que se viene recomendando en su aplicación desde sus orígenes (Lenz, Wells \& Kingston 2015). Otro de los elementos que son consustanciales al enfoque, es la horizontalidad en los procesos de trabajo, un espacio compartido de toma de decisiones y construcción de conocimiento que, a su vez, intersecciona con otra de las corrientes de apropiación y construcción del conocimiento como es la ciencia ciudadana, y más en su aproximación del diseño en la concepción de lo que algunos autores (Mueller et al. 2017) han denominado CDS (Citizen Design Science por sus siglas en inglés). Un término multidimensional que conceptualiza los aportes de Fueller a la ciencia del diseño y que junto con la aparición del concepto de ciencia ciudadana en los años noventa, los autores vienen a reconceptualizar en un campo aplicado al análisis de los sistemas urbanos. Desde esta mirada 
se añade la observación humana, la cognición de los sistemas, la experiencia, y el conocimiento de aporte local y abierto en la mejora de la planificación y el diseño de los espacios. En el contexto español, algunas son las experiencias que beben de los mismos principios y que se han venido en denominar diseño participativo en experiencias de ciencia ciudadana (Senabre, Ferran-Ferrer \& Perelló 2018). Ambas terminologías novedosas, y todavía por formalizar, sustentan en sus principios básicos el marco conceptual de la propuesta de este escrito al ser, una propuesta de diseño, que incide en aspectos de la planificación urbana, pero a través de una experiencia de ciencia ciudadana y que trata de generar conocimiento científico para buscar soluciones a un problema de salud pública. Se trata por tanto de un cambio en el paradigma en la generación de conocimiento que desde una visión tecnológica viene de la mano de la presencia de herramientas de democratización y acceso a las nuevas tecnologías de creación y fabricación digital (Gershenfeld 2012; Lee 2007). Acceso que está suponiendo la aparición de grandes espacios de intersección de las dimensiones del saber y nuevas vías de conocimiento y creación contemporánea. De esta forma parece presentarse un panorama que, bajo el paraguas metodológico STEAM, va a permitir abordar desde diferentes perspectivas e involucrando agentes con diferente nivel de implicación y profesionalización dichos procesos de co-creación. Propuesta que, en el ámbito de actuación del diseño pueden llegar a suponer, desde el análisis y la reflexión, poner en duda incluso el papel actual como creadores de los propios artistas y, diseñadores en este caso. Como algunos autores han apuntado (Manzini 2015), adoptarían la función de facilitadores de procesos de enseñanza, aprendizaje y formación creativo a nivel interno, pero sobre todo en lo que respecta a mediar en el aprendizaje en el resto de niveles educativos (Cambridge Assessment International Education, 2019) ${ }^{1}$ creando un modelo de educación integrada (Yakman 2008).

\section{OBJETIVOS Y DESCRIPCIÓN DEL PROYECTO}

En primer lugar, el proyecto nace con un objetivo general que involucra a todos los agentes, analizar la presencia de animales insectívoros en el entorno urbano. Hay que constatar que su ausencia parece relacionada con el aumento de insectos, convertido en un problema de salud pública. La finalidad, en el caso de la incorporación de nuestros estudiantes al proyecto, es la ideación de un artefacto que proporcione servicio a ese cometido general y en el que se 
van a involucrar los colegios de educación primaria. Desde el punto de vista formativo, se pretende que pongan en juego las múltiples competencias que involucran las distintas dimensiones del conocimiento que implica el proyecto: artística, tecnológica, científica y sociológica. Es este sentido, y desde una reflexión docente en el campo de la didáctica del diseño, cuando se valora el trabajo desde un enfoque STEAM. Una estrategia mucho más acorde que algunas de las estrategias proyectuales empleadas. De este modo, se plantea la necesidad de valorar su capacidad para contextualizar todos los elementos cognitivos presentes.

Se ha de apuntar que, la naturaleza del proyecto ha permitido que el artefacto ideado conjuntamente entre nuestros estudiantes y los centros de primaria, haya sido al mismo tiempo un prototipo para el diseño, desarrollo y puesta en marcha de una red IoT abierta a través de la comunidad y la plataforma, la red de las cosas Zaragoza (TTN Zaragoza por sus siglas en inglés). Un diseño, planificación y localización de antenas que se ha realizado en paralelo al resto de las etapas de diseño, pero que ha permitido al mismo tiempo, incorporar cierto conocimiento al bagaje formativo de nuestros estudiantes.

Ante los diversos aspectos que incorpora el proyecto, el escrito y los siguientes párrafos se centrarán en la descripción de la parte correspondiente al trabajo artístico y de diseño que compete a nuestros estudiantes en su interacción con los centros escolares de primaria.

En la propuesta, y durante el curso académico 2018/2019, han participado doce estudiantes del Grado en Diseño de Producto desde la asignatura de biónica. La propuesta comienza a formalizarse a partir de los diversos encuentros con los agentes involucrados y el correspondiente intercambio conocimiento transdisciplinar implicado en el problema. La fase de ideación parte de la premisa desarrollar un objeto artístico y de diseño funcional, que tuviera en cuenta sobre todo los elementos para la interacción y participación de los estudiantes de primaria. Elementos, como ya se ha señalado anteriormente, que abarcan desde la biología, las redes abiertas, la electrónica libre, sociología, educación y por supuesto lo que les es propio, el diseño. En las imágenes que se mostrarán a continuación se va recogiendo la evolución de distintas aportaciones, individuales y grupales, incorporando aspectos que provienen de los centros escolares, modificaciones consensuadas con otros agentes por la integración de los módulos de electrónica o los requerimientos de los sensores, etc. 
Los primeros prototipos virtuales diseñados por el alumnado (ver figura 1) toman en consideración y tras un proceso de indagación de los aspectos biológicos que además de las aves, en el entorno urbano se encuentran también salamandras (prototipo central) y murciélagos. Todas ellas especies vivas que se presentan como vectores de control de las plagas de insectos (mosquitos y mosca negra).
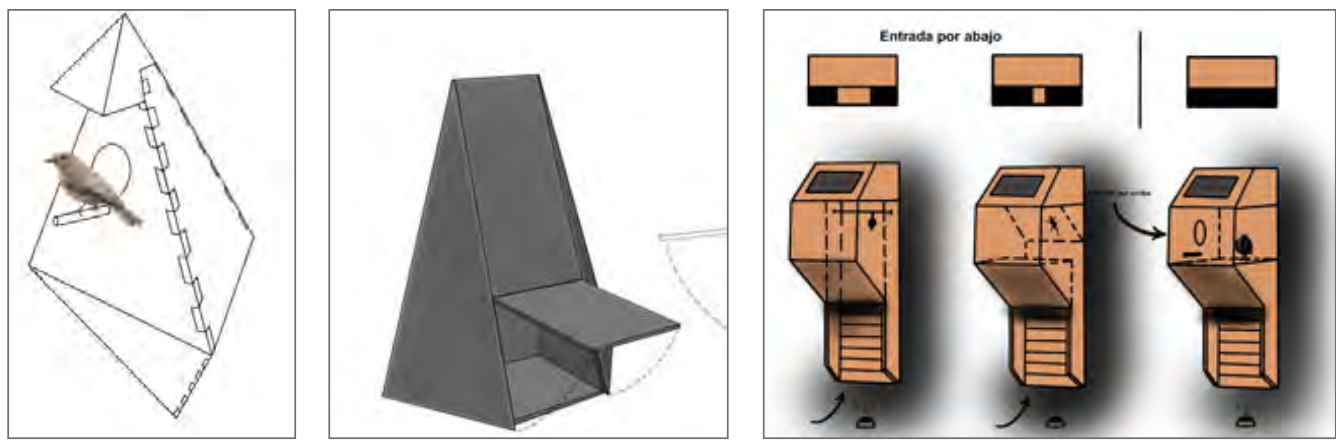

Figura 1: De izquierda a derecha distintos prototipos digitales, resolución de alojamiento de seres vivos insectívoros.

Prototipo derecha, versión final similar al prototipo funcional intervenido junto con estudiantes de primaria.

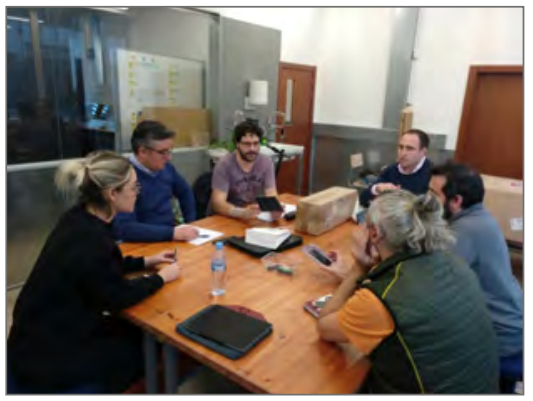

Es en esta etapa en la que se aprecia, además de lo señalado anteriormente, como se ponen en juegos los principos básicos STEAM, en los que el arte por un lado y las matemáticas por otro lado, van vehículando la planificación, organización y desarrollo de las fases y la evolución de la propuesta. En las figuras 2, 3, 4 y 5 se muestran distintos momentos que van, desde los primeros

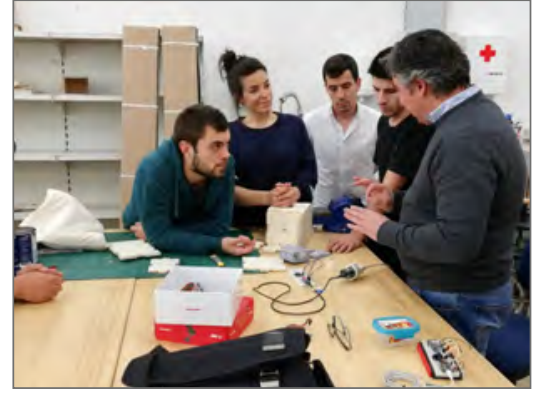

Figuras 2 y 3: Encuentro en la ESDA con expertos-voluntarios, y primer taller de creación electrónica arudino. encuentros en la ESDA (ver figura 2) hasta los talleres dirigidos por voluntarios expertos en IoT, hardware y software arduino (ver figura 3). El objetivo de estos encuentros y talleres era mejorar la competencia de nuestros estudiantes, pero ante todo que pusieran en juego elementos cognitivos que les facilitara la integración en sus propuestas de los diversos tipos de conocimiento. 
Hay que destacar que uno de los momentos calves es el encuentro entre los estudiantes y los maestros y estudiantes de educación primaria. En esa etapa es posible analizar qué tipos de competencias sociales y comunicativas, así procesos de interrelación se ponen en juego de cara a la cooperación horizontal con el contexto educativo señalado (ver figuras 4 y 5 ).
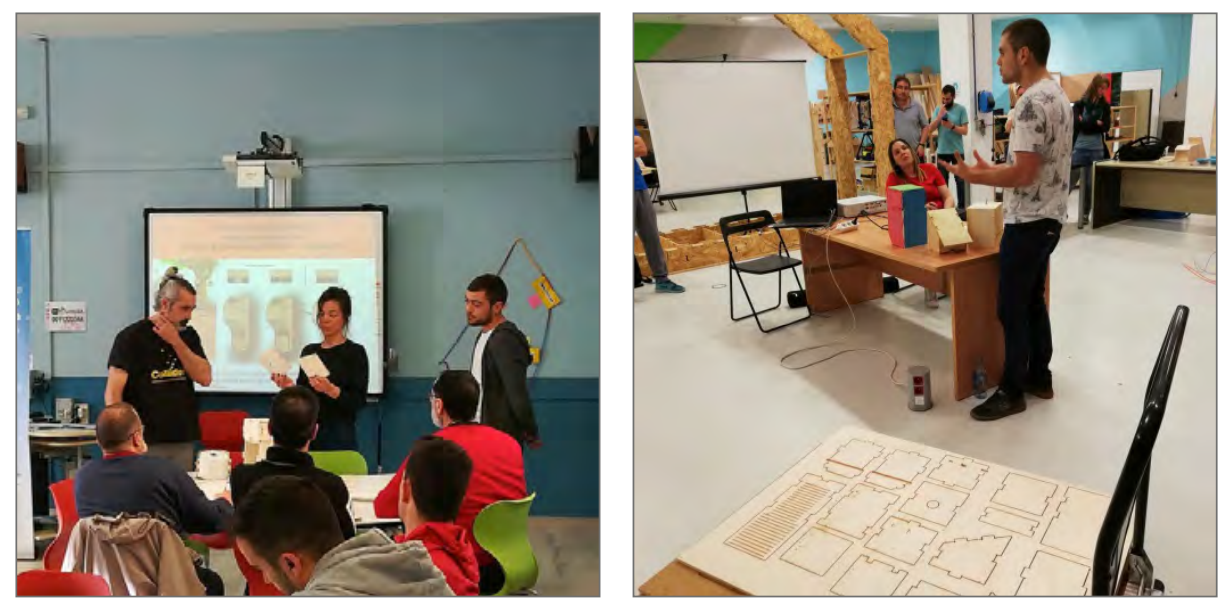

Figuras 4 (izquierda) y $\mathbf{5}$ (derecha). Estudiantes de diseño junto con el docente en el CIFE, centro de innovación y formación del profesorado (izquierda) y en el Centro de Arte y Tecnología (Etopía-CAT) mostrando el proyecto y el producto final a las maestras y maestros. Inferior, madera contrachapada sobre la que se troquelaron el conjunto de piezas que permitían el montaje de los distintos prototipos en función del ser vivo, decisión de los estudiantes de primaria.

En este sentido, y como se muestra en la parte inferior de la figura 5 , el proceso de ideación, diseño, producción, transporte elemento estandar en el que iban las piezas, y montaje debía ser flexible y ante todo coherente con las propuestas y las competencias y capacidad de los niños, asi como de los recursos de los centros escolares.

Un proceso en el que los estudiantes de primaria toman un protagonismo relevante, y en el que se realiza un trabajo compartido hasta la definición del elemento que se ha señalado en la figura anterior.

Hay que destacar que esta última fase, en la que los estudiantes de diseño comparten sus conoci-

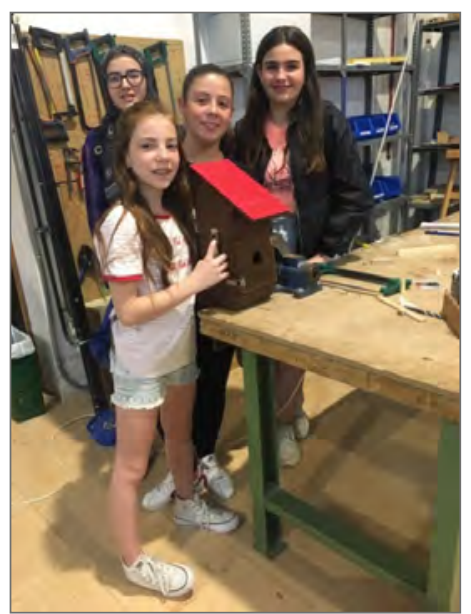

Figuras 6: Imagen izquierda, alumnas de un Centro escolar de Fuentes de Ebro (Zaragoza) que, junto con el docente y tras un trabajo previo sobre el prototipo de diseño trabajaron en una adaptación a su contexto escolar, así cómo en la intervención plástica. 


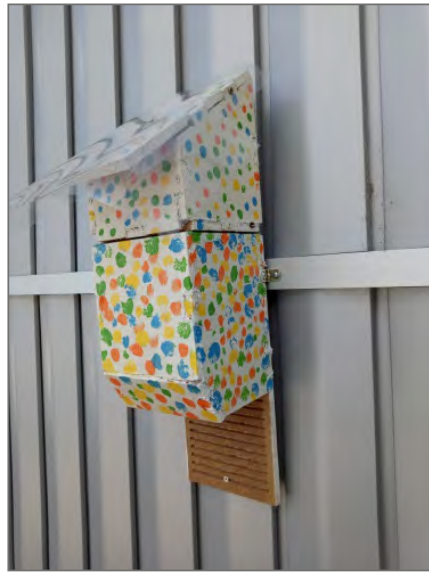

Figura 7: Casetas de pájaros intervenidas y rediseñadas por estudiantes de primaria en los talleres artísticos y de diseño en colaboración con nuestros estudiantes. En la figura de la derecha caseta colocada en la fachada del CEIP Lés Allées-Vadorrey (Colegio de eduación infantil y primaria). mientos con estudiantes de primaria, resulta la más interesante de cara al análisis, dado que en ella se puede comprobar cómo se ha ido implementando cada una de las etapas del proyecto en su propia concepción STEAM. Se puede valorar, qué conocimientos científicos, tecnológicos y de diseño han incorporado nuestros estudiantes, cómo los comparten y transfieren, con la finalidad de desarrollar, de forma conjunta una nueva dimensión artística y de diseño a otro nivel educativo. Una fase en la que, pasan de tener un papel de meros diseñadores, a co-creadores, junto con los estudiantes de primaria, siempre desde el respeto entre iguales en un contexto abierto de enriquecimiento mutuo, y que se ha expresado de forma repetida por ambas partes.

\section{RESULTADOS: ELEMENTOS PARA LA REFLEXIÓN Y CONCLUSIONES}

El proyecto, como hemos ido apuntando a lo largo de los apartados anteriores, presenta muchos elementos para el análisis. De dichos elementos cabe destacar el análisis de la participación de los distintos agentes involucrados en la consecución del objetivo general del proyecto y cómo se ponen de manifiesto en el marco del enfoque STEAM.

En todo caso, y por la finalidad de este artículo, este apartado se centrará en valorar y analizar los resultados que atienden a los logros de nuestros estudiantes de diseño. En dicho análisis se han empleado, desde un marco cualitativo, técnicas de corte etnógrafico, a partir del análisis de la información recogida en sus memorias de proyecto, así como el cuaderno de campo y el desarrollo de un grupo de discusión. Una información que ante todo se sustenta en el análisis del discurso realizado por el docente responsable del proyecto y de este artículo.

Se parte de la base de un objetivo inicial planteado a nuestros estudiantes, la ideación de un dispositivo que no solo fuera funcional, sino que incorporara los 
elementos que les son propios a su formación como diseñadores, y entre los que destacan las características estéticas, visuales y plásticas del artefacto. A partir de ese hecho se valora como poner en juego todos los elementos, y como en el marco de desarrollo de la metodología STEAM, se incorporan muchos otros aspectos por su valor cognitivo. En este sentido los estudiantes señalaron que, a pesar de las dificultades iniciales para compatibilizar la información de los expertos, e integrarlas en la fase inicial de ideación de su dispositivo, la metodología les ha permitido movilizar conocimientos y competencias básicas de su formación, aplicándolas a un problema real. Competencias que están vinculadas con su capacidad creativa, el desarrollo cognitivo en cuanto a la comprensión de procesos, así como contextos diversos, y ante todo la dimensión social que pone en juego el trabajo en una propuesta con tantos agentes involucrados.

Uno de los aspectos más relevantes que han destacado es como en muchas de las fases del desarrollo proyecto, han adoptado otros papeles que no se acomodaban a su objetivo inicial. Los estudiantes apuntan a como han movilizado habilidades para la comunicación y la facilitación de procesos entre los distintos agentes y en muchas ocasiones actuando como articuladores en el desarrollo de la propia propuesta.

Si atendemos a los aspectos más 'creativos', de los aspectos destacados a partir del análisis de sus memorias, y de lo reflejado en el grupo de discusión, ellos señalan a la necesidad de explicar de forma continuada el diseño en sí, más allá de cuestiones funcionales, aspectos que no siempre eran atendidos y entendidos por agentes provenientes de las dimensiones más tecnológicas. Esto les ha supuesto en muchas ocasiones corresponsabilizarse de la toma de decisiones que les permitiera integrar de forma adecuada todas las sensibilidades.

Para finalizar, los estudiantes destacaron las etapas en las que ante todo han trabajado en el contexto educativo de los centros de enseñanza primaria. Les ha supuesto comprender a los usuarios participantes en un trabajo entre iguales en el que, sus ideas y conceptos iniciales para el diseño del artefacto, eran recogidas por los maestros y estudiantes en un trabajo compartido.

De esta forma se puede decir que, la propuesta les ha llevado a movilizar múltiples competencias y habilidades en la resolución, no solo de un problema afín a su dimensión de diseño, sino al resto de dimensiones e intervinientes presentes. De esta forma, y en coherencia con lo señalado por autores des- 
tacados en el análisis del potencial de la metodología (Maeda 2013; Yakman 2008), para el aprendizaje, los aspectos cognitivos relacionados con la dimensión artística, son capaces de movilizar muchas otras competencias, como así podemos afirmar en el caso presentado.

Por otra parte, y como en el escrito se ha puesto de manifiesto, estas propuestas parecen insertarse en el marco de un cambio de paradigma social donde el conocimiento deviene más horizontal, democrático y coparticipado en la búsqueda y solución de problemas y planteamiento de experiencias (Müeller et al. 2017; Senabre et al. 2018). Un cambio facilitado por la presencia de herramientas abiertas (IOT, arduino, herramientas de fabriación digital) que facilitan y ponen en valor la interacción y la integración de conocimientos de distintas personas, con diferente nivel de profesionalización e intereses (Gershenfeld 2012; Lee 2007). En estos escenarios, que ponen en juego múltiples capacidades y competencias, el arte y el diseño, integrado como dimensiones vehiculares en el enfoque STEAM permiten que el pensamiento creativo transforme el hacer de nuestros estudiantes en agentes activos, capaces de intervenir en su realidad social (Azkaray 2019).

En conclusión, y a falta del desarrollo y análisis de otras propuestas en curso, se trata de una metodología que en este caso, y por su carácter proyectual, así como por su propio origen en una escuela de diseño, parece adecuarse a los intereses y a la movilización de las distintas dimensiones que vinculan los aspectos cognitivos 'intelectuales' y creativos.

\section{Referencias bibliográfícas}

Azcaray Fernández, Joseba Koldobika. 2019. "Metodología para integrar el diseño en un proceso curricular STEAM a través del uso de las nuevas tecnologías creativas". Tesis Univ. Politècnica de València. http://hdl.handle.net/10251/125704

Gershenfeld, Neil. 2012. "How to make almost anything: The digital fabrication revolution". Foreign Affairs 91(6): 42-57

Lee, Yanki. 2007. "Design participation tactics: Involving people in the design of their built environment". Tesis Hong Kong Polytechnic Univ. http://ira.lib.polyu.edu.hk/handle/10397/2255

Lenz, Bob. 2015. Transforming schools using project-based learning, performance assessment, and common core standards. With Justin Wells \& Sally Kingston. San Francisco CA: Jossey-Bass

Maeda, John. 2013. "STEM + Art = STEAM". The STEAM Journal 1(34): 1-3. https://doi. org/10.5642/steam.201301.34 
Manzini, Ezio. 2015. Design, when everybody designs: An introduction to design for social innovation. Translated by Rachel Coad. Boston MA: MIT

Mueller, Johannes et al. 2017. "Citizen design science: A strategy for crowd-creative urban design". Cities 72: 181-8. https://doi.org/10.1016/j.cities.2017.08.018

Senabre Carbonell, Enric, Núria Ferran Ferrer \& Josep Perelló Mulet. 2018. "Diseño participativo de experimentos de ciencia ciudadana". Comunicar 54: 29-38. https://recyt.fecyt.es/ index.php/comunicar/article/view/62675

University of Cambridge Local Examinations Syndicate (UCLES). 2019. "Cambridge Primary Art \& Design (0067)". Subject programme. https://www.cambridgeinternational.org/programmes-and-qualifications/cambridge-primary/curriculum/art-and-design

Yakman, Georgette. 2008. "STEAM Education: An overview of creating a model of integrative education". Tesis Virginia Polytechnic and State Univ.

\section{Notas}

1 Cambridge Assessment International Education 2019. https://www.cambridgeinternational. org/programmes-and-qualifications/cambridge-primary/curriculum/art-and-design 